\title{
Transient infrared thermography to characterize thermal properties of millimeter- sized low conductivity materials
}

\author{
by T. Pierre, M. Carin, M. Courtois, P. Carré
}

\author{
Univ. Bretagne Sud, UMR CNRS 6027, IRDL, F-56100 Lorient, France, thomas.pierre@univ-ubs.fr
}

\begin{abstract}
This work deals with the thermal characterization of a low conductivity material of millimeter-sized used mainly in building insulation, the hemp shiv. Experimentally, the samples are positioned in a stainless steel chamber, in which it is possible to control the environment. They are heated during few seconds on one side and an infrared camera measures the temperature field of the sample surface. The experimental thermograms are compared with a theoretical estimation model derived from the fin model in order to estimate simultaneously two parameters by ordinary least-squares regression: the thermal diffusivity and a modified Biot number.
\end{abstract}

\section{Introduction}

The development of accurate and simple experiments dedicated to the thermal characterization of millimetersized materials is of great importance. For example, in the domain of building, there is a lack of knowledge concerning the thermal properties of hemp shiv, one of the component of hemp concrete. Hemp concrete, increasingly used, is a porous heterogeneous material composed of millimeter-sized hemp shives mixed in a lime binder. With appropriate proportions, it can cover different uses in the building: roof insulation, wall and ground floor insulating slabs [1]. Many studies concern, on the one hand, the characterization and the behavior of the hemp concrete versus temperature and humidity and, on the other hand, the development of predictive numerical models, which need these data [2]. If the physical properties of the binder can easily be measured, it is not the case for the hemp shives. To our knowledge, the only available data concern the estimation of an effective thermal conductivity of hemp shives in bulk through the hot-wire technique [3]. There is no direct experimental measurement of the hemp shiv itself. However, literature mentions the estimation of an effective conductivity by inverse method between experimental results and a complete numerical model of the hemp concrete considering the size and the orientation of the hemp shives [2]. However, these estimations have to be checked with direct experimental measurements.

The thermal characterization lays on the fitting, for example, of a model giving theoretical temperatures to experimental data. One of the difficulties is to know precisely the experimental boundary conditions in order to have a direct model as faithful as possible with the experiment. To circumvent this problem, Rémy et al [4] propose a methodology to estimate the thermal diffusivity of a glass plate during transient experiments. They measure the temperature field on the surface of the sample heated on one side versus time using an infrared camera. Then, based on experimental thermograms measured at three coordinates $z_{,} z_{1}$, and $z_{2}$, the temperature at the $z$ coordinate is expressed as a linear function of the temperatures at the $z_{1}$ and $z_{2}$ coordinates situated on either side of $z$. Thus, the estimation of the thermal diffusivity is made possible by fitting the theoretical temperature expressed at the $z$ location on the experimental one. Therefore, the knowledge of the boundary conditions is not necessary.

The technique proposed by Rémy et al has been adapted in our case. This work proposes to estimate the thermal diffusivity of millimeter-sized anisotropic hemp shives when the heat flux is parallel and orthogonal to their fibers. At first, the methodology of estimation is tested on known low conductivity materials, polycarbonate and anisotropic balsa. Since there are convective heat losses along the sample, the theoretical model is based on the fin model. Consequently, there is a second parameter to estimate, a modified Biot number, which includes a heat exchange coefficient. Experimentally, the influence of the surrounding environment is also analyzed with measurements performed in vacuum, natural convection, and forced convection.

\section{Theoretical considerations}

\subsection{The theoretical model}

As depicted in Fig. 1, a heat flux is applied on the top surface of the sample. Heat losses are considered on the other surfaces. In the fin model, only heat transfer along the descendent $z$ direction is taken into account. The heat equation is then given by Eq. (1), where $\bar{T}(z, t)$ is the average temperature of the cross section at the location $z, k_{z}$ and $\alpha_{z}$ respectively the thermal conductivity and diffusivity of the sample, $h$ the heat exchange coefficient, and $e$ and $/$ are the sample thickness and width, respectively. 


$$
\frac{\partial^{2} \bar{T}(z, t)}{\partial z^{2}}-\frac{2 h(e+l)}{e l} \frac{\bar{T}(z, t)}{k_{z}}=\frac{1}{\alpha_{z}} \frac{\partial \bar{T}(z, t)}{\partial t}
$$

The Eq. (1) is easily solved using the Laplace transform and the quadrupole formalism [5]. Since the boundary conditions cannot be well-known precisely, the method proposed by Rémy and al. [4] considers a linear combination between the temperature at location $z$ with temperatures located at the positions $z_{1}$ and $z_{2}$ on either side of $z$ as presented in Eqs. (2)-(6), where $s$ is the Laplace parameter.

$$
\begin{aligned}
\bar{T}(z, t)=\bar{T}\left(z_{1}, t\right) \otimes L^{-1}\left[F_{1}(z, s)\right]+\bar{T}\left(z_{2}, t\right) \otimes L^{-1}\left[F_{2}(z, s)\right] \\
F_{1}(z, s)=\frac{\sinh \left[\gamma\left(z_{2}-z\right)\right]}{\sinh \left[\gamma\left(z_{2}-z_{1}\right)\right]} \\
F_{2}(z, s)=\frac{\sinh \left[\gamma\left(z-z_{1}\right)\right]}{\sinh \left[\gamma\left(z_{2}-z_{1}\right)\right]} \\
\gamma=\sqrt{\frac{s}{\alpha_{z}}+H_{z}} \\
H_{z}=\frac{h(e+l)}{k_{z} e l}
\end{aligned}
$$

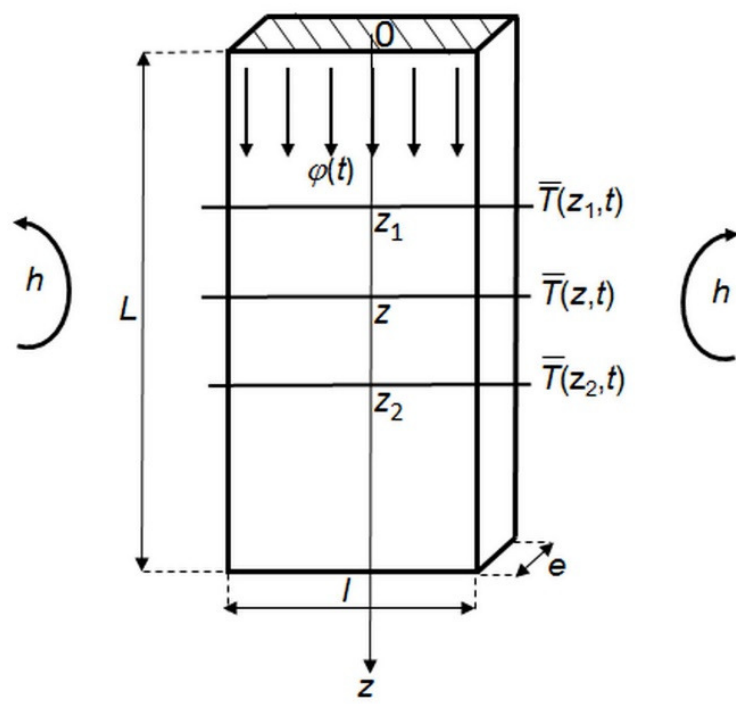

Fig. 1: sketch of the theoretical model of the fin.

Actually, the temperatures $\bar{T}\left(z_{1}, t\right)$ and $\bar{T}\left(z_{2}, t\right)$ are the experimental ones obtained through infrared thermography at the locations $z_{1}$ and $z_{2}$, respectively. The theoretical temperature $\bar{T}(z, t)$ in Eq. (2) is fitted with experimental temperature $\bar{T}^{\exp }(z, t)$ by ordinary least-squares regression coupled with a Levenberg-Marquardt algorithm by estimating the thermal diffusivity $\alpha_{z}$ and the modified Biot number $H_{z}$, by solving the criteria (7) where $\beta$ represents the parameters and $N$ the number of points:

$$
\sum_{i=1}^{N}\left|\bar{T}\left(z, t_{i}\right)-\bar{T}^{\exp }\left(z, t_{i}, \beta\right)\right|^{2} \rightarrow 0
$$

Finally, the thermal conductivity and the heat exchange coefficient can then be deduced from measurements of the heat capacity and density obtained by appropriate apparatus. 


\subsection{The sensitivity study}

The possibility to estimate both the thermal diffusivity and the modified Biot number lays on the sensitivity analysis of the temperature $\bar{T}(z, t)$ with these parameters. The reduced sensitivity $(8)$ is plotted in Figs. 2 and 3 for low conductivity materials: polycarbonate (left) and balsa (right).

$$
X_{\beta}=\beta \frac{\partial T(z, t)}{\partial \beta}
$$

The properties used for the simulation are those found in the literature [6-7]:

- Polycarbonate: $k=0.200 \mathrm{~W} \cdot \mathrm{m}^{-1} \cdot \mathrm{K}^{-1}, \rho=1000 \mathrm{~kg} \cdot \mathrm{m}^{-3}, c_{p}^{m}=1250 \mathrm{~J} \cdot \mathrm{kg}^{-1} \cdot \mathrm{K}^{-1}$;

- Balsa: $k=0.05 \mathrm{~W} \cdot \mathrm{m}^{-1} \cdot \mathrm{K}^{-1}, \rho=100 \mathrm{~kg} \cdot \mathrm{m}^{-3}, c_{p}^{m}=1400 \mathrm{~J} \cdot \mathrm{kg}^{-1} \cdot \mathrm{K}^{-1}$.

Then tests are performed for the three types of surrounding conditions:

- Vacuum: $h=2 \mathrm{~W} \cdot \mathrm{m}^{-2} \cdot \mathrm{K}^{-1}$ (close to $4 \varepsilon \sigma T^{3}$ );

- Natural convection: $h=10 \mathrm{~W} \cdot \mathrm{m}^{-2} \cdot \mathrm{K}^{-1}$;

- Forced convection: $h=40 \mathrm{~W} \cdot \mathrm{m}^{-2} \cdot \mathrm{K}^{-1}$.

Results are presented in Figs. 2 and 3 for a random position $z_{1}=2 \mathrm{~mm}, z=3 \mathrm{~mm}$, and $z_{2}=5 \mathrm{~mm}$. The solid lines concern the temperature sensitivity to the thermal diffusivity and the dashed line to the modified Biot number. In each case, it is shown that the estimation of both parameters is possible. As expected, the temperature becomes less sensitive to the modified Biot number, when the latter decreases.

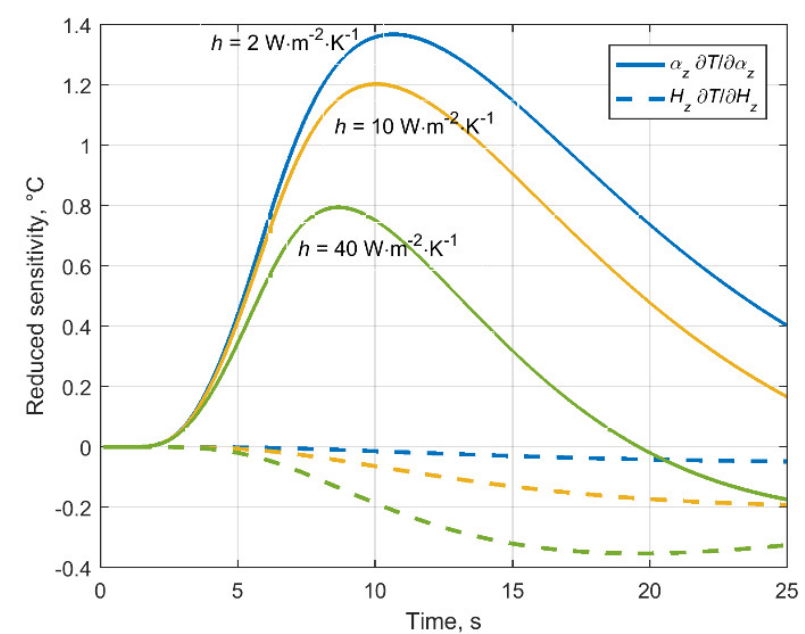

Fig. 2: Reduced temperature sensitivities for the thermal diffusivity and the modified Biot number versus time obtained for polycarbonate for three types of environment condition.

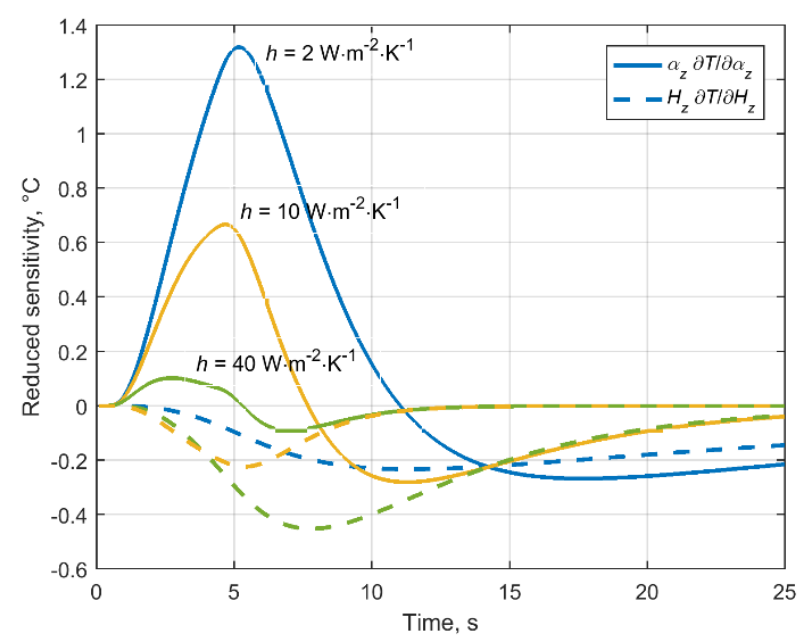

Fig. 3: Reduced temperature sensitivities for the thermal diffusivity and the modified Biot number versus time obtained for balsa for three types of environment condition.

\section{The experimental apparatus}

Figs. 4 and 5 present a global view of the experimental apparatus composed of a stainless steel chamber with a sapphire window in which the heated sample is placed and of an IR camera. The vacuum can be performed within the chamber. The IR camera is a Flir X6580sc model working in the range $[1.5 \mu \mathrm{m}-5.1 \mu \mathrm{m}]$ and equipped with a $15 \mu \mathrm{m}$ resolution macroscopic lens. The acquisition frequency is $50 \mathrm{~Hz}$ and the tests last $30 \mathrm{~s}$. As drawn in Fig. 6, the sample is warmed with a heating element covered by a thin aluminum foil, on which the test sample is stuck. The aluminum foil is used to avoid the radiation of the heating element to the sample. A type $\mathrm{K}$ thermocouple is placed at the rear side of the sample only to control initially the temperature given by the camera, then it is removed. The heating is considered as a crenel stopped when the sample temperature increases a maximum of about $20^{\circ} \mathrm{C}$. Consequently, the sample emissivities do not need to be known since they can be considered as a proportionality constant. Three types of surrounding are considered in the stainless steel chamber: vacuum, natural convection, and forced convection. It is noteworthy that the air velocity is not measured and the Reynolds number cannot be calculated. 
Five samples of different materials are tested. Here are their sizes and weight:

- Isotropic polycarbonate: $L=(20.33 \pm 0.01) \mathrm{mm}, \quad I=(10.12 \pm 0.01) \mathrm{mm}, \quad e=(1.98 \pm 0.01) \mathrm{mm}$, $m=(0.449 \pm 0.001) \mathrm{g}$.

- Balsa with fibres orthogonal to the heat flux $(\perp): L=(15.48 \pm 0.01) \mathrm{mm}, I=(6.90 \pm 0.01) \mathrm{mm}$, $e=(1.68 \pm 0.01) \mathrm{mm}, m=(0.038 \pm 0.001) \mathrm{g}$.

- Balsa with fibres parallel to the heat flux $(/ /): L=(16.19 \pm 0.01) \mathrm{mm}, I=(6.43 \pm 0.01) \mathrm{mm}, e=(1.82 \pm 0.01) \mathrm{mm}$, $m=(0.033 \pm 0.001) \mathrm{g}$.

- Hemp shiv with fibres orthogonal to the heat flux $(\perp): L=(5.60 \pm 0.001) \mathrm{mm}, I=(15.17 \pm 0.01) \mathrm{mm}$, $e=(1.80 \pm 0.01) \mathrm{mm}, m=(0.031 \pm 0.001) \mathrm{g}$.

- Hemp shiv with fibres parallel to the heat flux $(/ /): L=(17.62 \pm 0.01) \mathrm{mm}, I=(6.03 \pm 0.01) \mathrm{mm}$, $e=(0.97 \pm 0.01) \mathrm{mm}, m=(0.028 \pm 0.001) \mathrm{g}$.

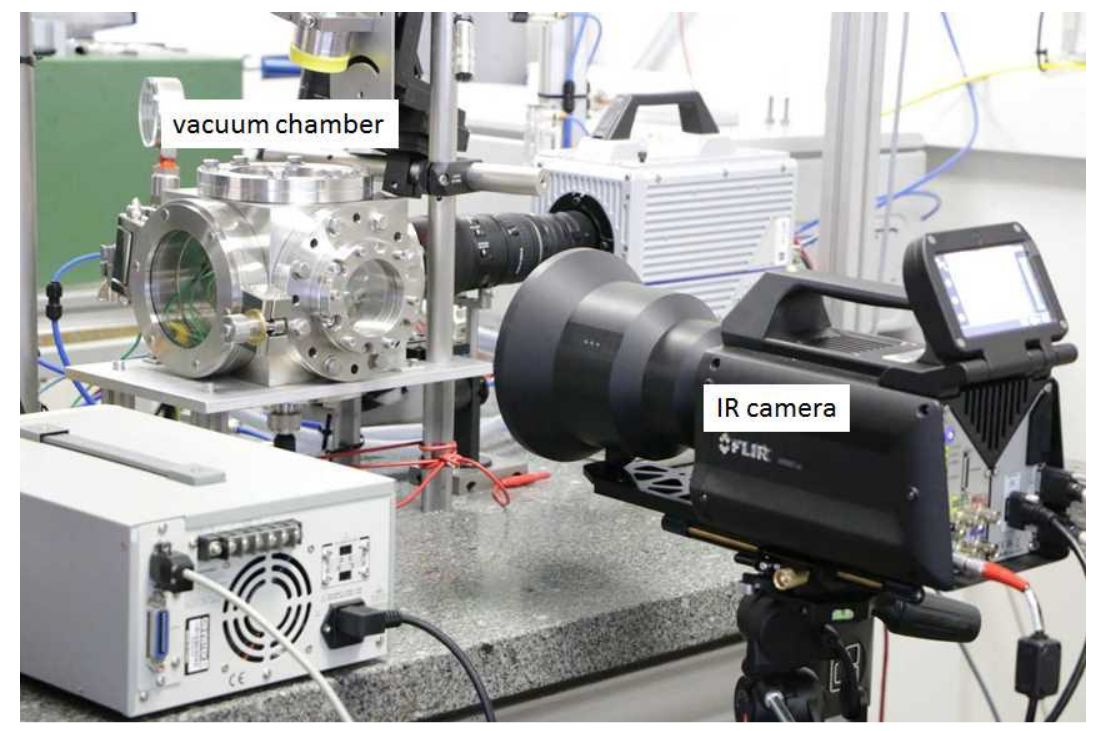

Fig. 4: Experimental apparatus: the vacuum chamber and the IR camera.

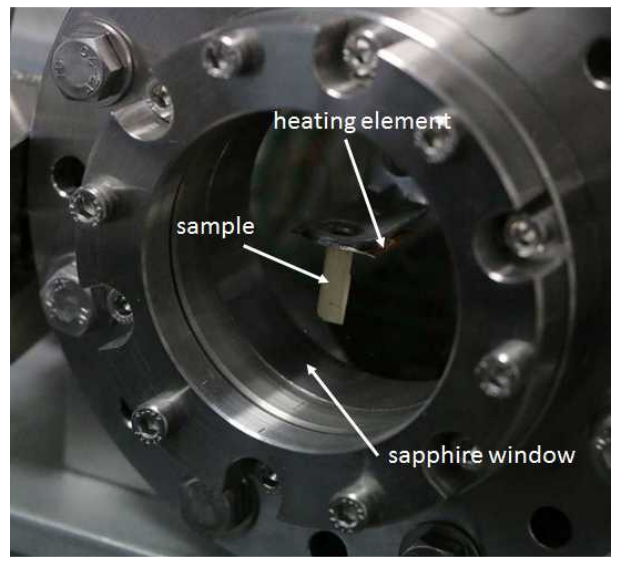

Fig. 5: Experimental apparatus: zoom of the sample and the heating element.

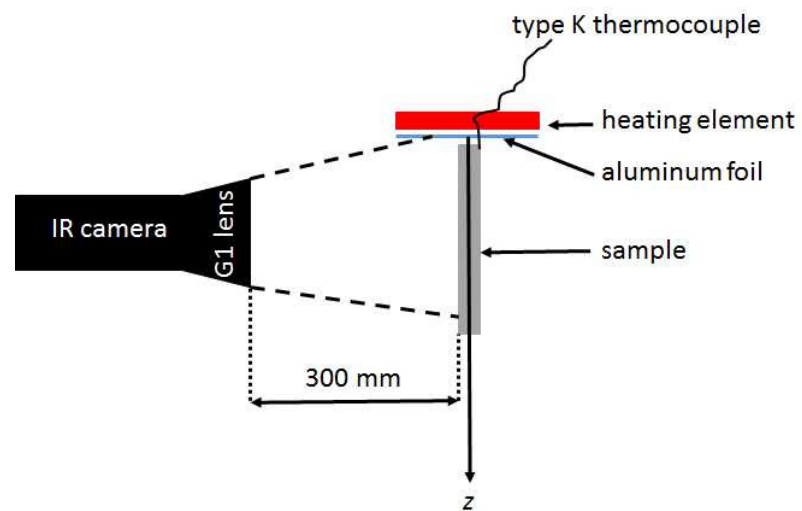

Fig. 6: Sketch of the experimental apparatus.

\section{Results and discussion}

Fig. 7 shows an example of IR image of the $\perp$ balsa sample with five lines. The average temperature of each line is recorded versus time. Thus, according to the theoretical methodology developed in section 2, there are ten possible combinations with these five average temperatures. 


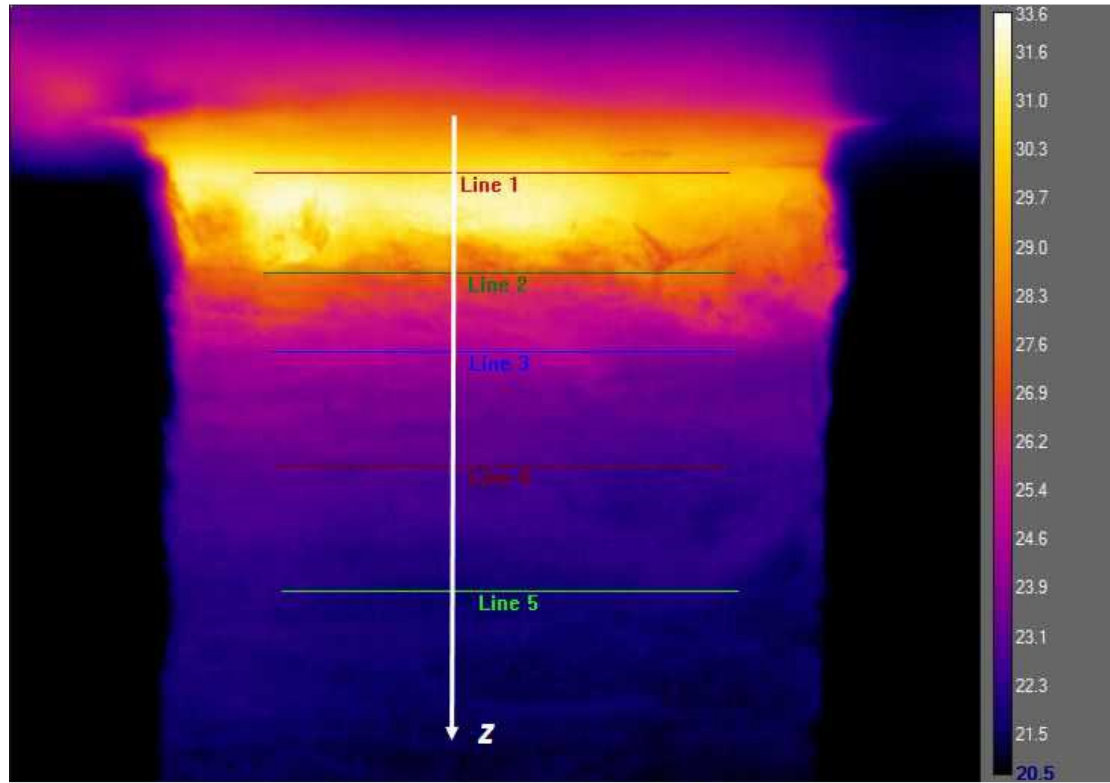

Fig. 7: Example of IR thermogram for $\perp$ balsa. The temperature scale shown on the right is expressed in degree Celsius.

In Fig. 7, the five lines are located at the positions $z=0,0.99 \mathrm{~mm}, 1.77 \mathrm{~mm}, 2.90 \mathrm{~mm}$, and $4.14 \mathrm{~mm}$. Fig. 8 plots both the evolutions of the five average temperatures (solid lines) at these locations and their temperature derivatives (dashed lines) versus time:

$$
\frac{\partial T(z, t)}{\partial t}
$$

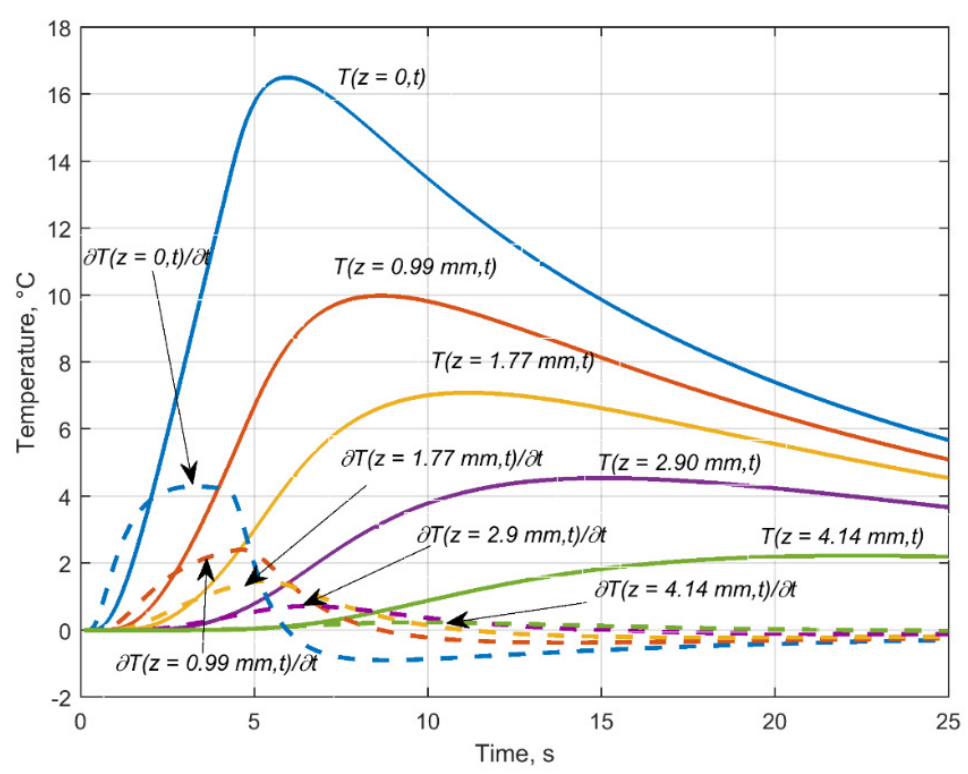

Fig. 8: Evolution of the temperatures and their time derivatives at the z-positions versus time.

It is shown that the derivatives decrease when the z-position increases as expected. Indeed, the temperature is less sensitive to the thermal diffusivity if the derivative becomes null, which is almost the case for the temperature $T(z=4.14 \mathrm{~mm}, t)$. However, for three temperatures with high enough time derivatives (9), the plot shows that the estimation time must last until the second time derivative $\partial \bar{T}(z=4.14 \mathrm{~mm}, t) / \partial t$ reaches its maximum.

The quality of the estimation is also discussed through the calculation of the covariance $\sigma_{\alpha_{z} H_{z}}$ from the covariance matrix (10) and of the residuals. 


$$
\left[\begin{array}{cc}
\sigma_{\alpha_{z}}^{2} & \sigma_{\alpha_{z} H_{z}} \\
\sigma_{\alpha_{z} H_{z}} & \sigma_{H_{z}}^{2}
\end{array}\right]
$$

Table 1 gathers estimation results for the ten possible combinations. The heat exchange coefficient is given instead of the modified Biot number using Eqs. (6) and (11).

$$
\alpha_{z}=\frac{k_{z}}{\rho c_{p}^{m}}
$$

The convergence is not achieved for the four last combinations, due to null time derivative of the temperature. The six first cases are successful, the covariance between the thermal diffusivity and the heat exchange coefficient is close to zero, which means that both parameters can be estimated separately. The experiment is performed in vacuum. Consequently, the valuable estimation of the heat exchange seems to be for the combinations 2 and 3 , where both the covariances are weak. In the vacuum, the theoretical linearized heat exchange coefficient can be evaluated at $h^{\text {th }}=3.3$ $\mathrm{W} \cdot \mathrm{m}^{-2} \cdot \mathrm{K}^{-1}$ using the following expression:

$$
h^{\text {th }}=4 \varepsilon \sigma T^{3}
$$

where $\varepsilon$ is the emissivity of the balsa (here 0.63 ) and $\sigma=5.67 \times 10^{-8} \mathrm{~W} \cdot \mathrm{m}^{-2} \cdot \mathrm{K}^{-4}$. Thus, the thermal diffusivity can be estimated considering results from combinations 2 and 3 .

Table 1: Estimation results for all the possible combinations in the case of $\perp$ balsa.

\begin{tabular}{c|c|c|c|c|c|c} 
combination & $z_{i} / z^{\prime} z_{\mathrm{j}}$ & convergence & $\sigma_{\alpha_{z} H_{z}}$ & $\alpha_{k}\left(\mathrm{~mm}^{2} \cdot \mathrm{s}^{-1}\right)$ & $h\left(\mathrm{~W} \cdot \mathrm{m}^{-2} \cdot \mathrm{K}^{-1}\right)$ & $\begin{array}{c}\text { covariance matrix } \\
\text { of residuals }\end{array}$ \\
\hline 1 & $0 / 0.99 / 1.77$ & yes & 0.047 & 0.304 & 0.7 & 0.022 \\
\hline 2 & $0 / 0.99 / 2.90$ & yes & -0.0008 & 0.407 & 2.9 & 0.032 \\
\hline 3 & $0 / 0.99 / 4.14$ & yes & 0.104 & 0.467 & 3.7 & 0.017 \\
\hline 4 & $0 / 1.77 / 2.90$ & yes & 0.115 & 0.228 & 9.4 & 0.197 \\
\hline 5 & $0 / 1.77 / 4.14$ & yes & 0.097 & 0.378 & 9.2 & 0.185 \\
\hline 6 & $0 / 2.90 / 4.14$ & yes & 0.125 & 0.319 & 10.9 & 0.230 \\
\hline 7 & $0.99 / 1.77 / 2.90$ & no & 1 & $\mathrm{x}$ & $\mathrm{x}$ & $\mathrm{x}$ \\
\hline 8 & $0.99 / 1.77 / 4.14$ & no & 1 & $\mathrm{x}$ & $\mathrm{x}$ & $\mathrm{x}$ \\
\hline 9 & $0.99 / 2.90 / 4.14$ & no & 1 & $\mathrm{x}$ & $\mathrm{x}$ & $\mathrm{x}$ \\
\hline 10 & $1.77 / 2.90 / 4.14$ & no & 1 & $\mathrm{x}$ & $\mathrm{x}$ & $\mathrm{x}$
\end{tabular}

Fig. 9 presents the estimation plots for the combination 3. The upper figure plots the temperature evolutions versus time and the residuals. The lower figure plots the reduced sensitivities of the estimated temperature $T(z, t)$ versus the estimated parameters. As previously shown in Fig. 2 , in vacuum, the sensitivity of the temperature to the heat exchange coefficient is very weak compared to the sensitivity to the thermal diffusivity.

The last point concerns the estimation time. Figs. 10,11, and 12 plot the evolution of the estimation of the thermal diffusivity and of the heat exchange coefficient versus the estimation time. Obviously, it is necessary to have a minimum time of estimation, since estimated values tend to a constant one. In Figs. 10, 11, and 12, a minimum estimation time of $20 \mathrm{~s}$ is required. This observation can be related to the sensitivity of the time derivative (9), which tends to zero after $10 \mathrm{~s}$ after reaching its maximum.

The present methodology is applied to the five samples presented in section 3 for three kinds of environment conditions in the chamber. The estimation results of the polycarbonate and of the balsa samples are compared with some found in the literature. And then, two hemp shiv samples are characterized. 
$\alpha_{z}=0.467 \mathrm{~mm}^{2} \cdot \mathrm{s}^{-1} ; h=3.7 \mathrm{~W} \cdot \mathrm{m}^{-2} \cdot \mathrm{K}^{-1} ; \mathrm{y} 1=0 \mathrm{~mm} ; \mathrm{y}=0.99 \mathrm{~mm} ; \mathrm{y} 2=4.14 \mathrm{~mm}$
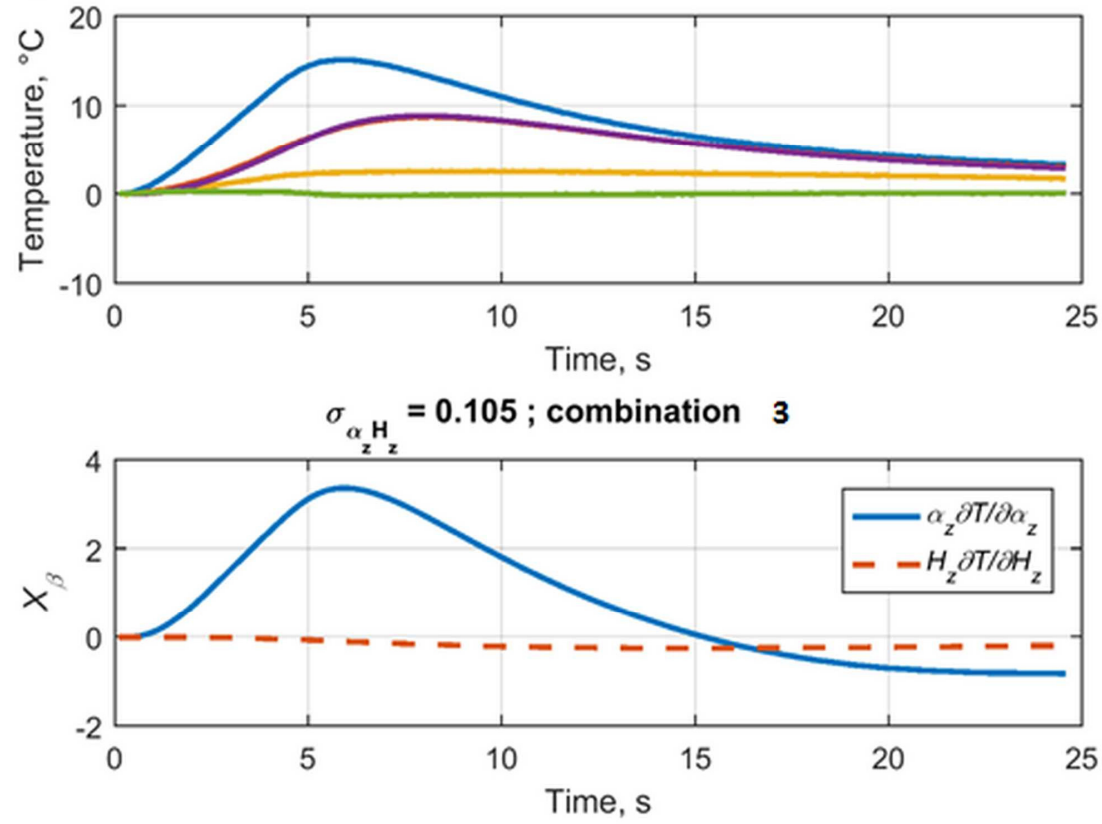

Fig. 9: Example of estimation and results in the case of the balsa $\perp$ in vacuum.

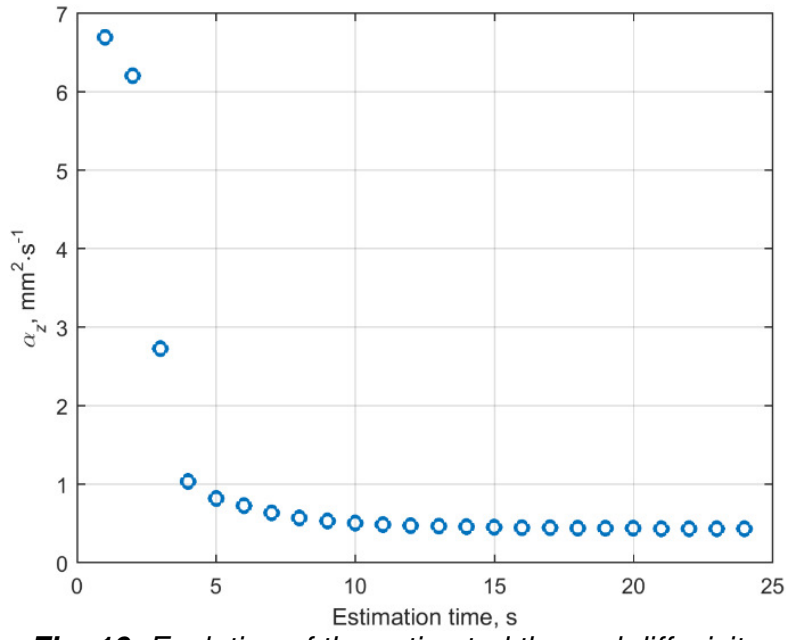

Fig. 10: Evolution of the estimated thermal diffusivity versus the estimation time in the case of the balsa $\perp$ sample during experiment in the vacuum.

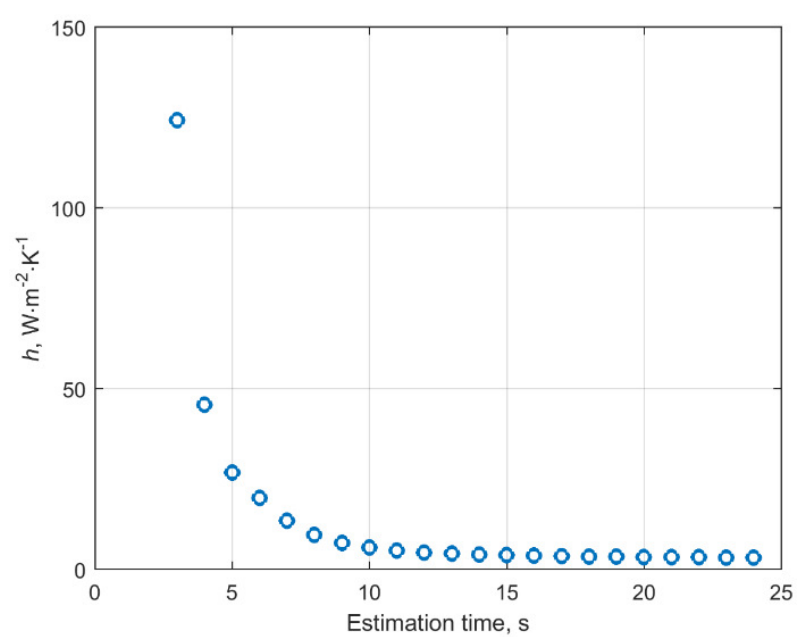

Fig. 11: Evolution of the estimated heat exchange coefficient versus the estimation time in the case of the balsa $\perp$ sample during experiment in the vacuum. 


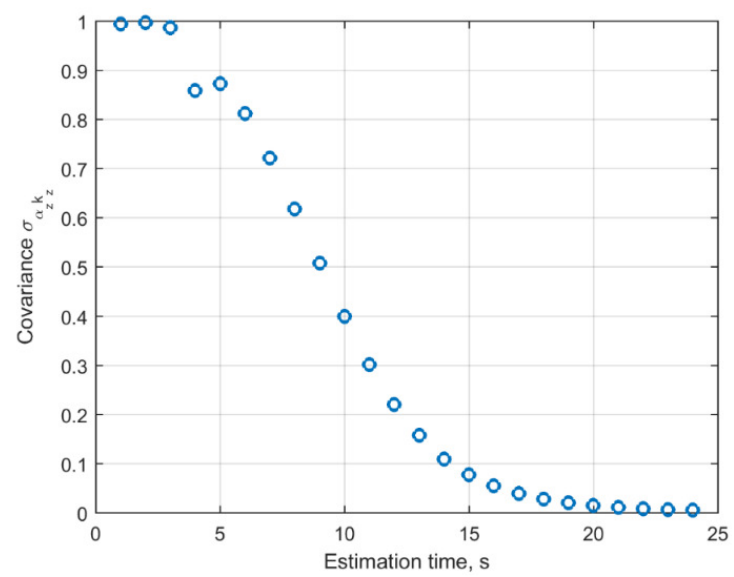

Fig. 12: Evolution of the covariance versus the estimation time in the case of the balsa $\perp$ sample during experiment in the vacuum.

Table 2 gathers all estimation results for the five samples. The estimation of the thermal diffusivity of polycarbonate leads to an average value of the same order of magnitude but higher than those found in the literature of $28 \%$ [7]. The estimation of the balsa thermal diffusivities shows a ratio of about 2.2 between the perpendicular and the orthogonal samples, which is consistent with results found in the literature $[6 ; 8]$. The same observation can be done for the hemp shives, which values are also consistent with those found in the literature for cellulosic materials [8]. Most of the time, the covariance matrix tends to a null value, which makes us considering that both parameters are uncorrelated and have been estimated properly. The only cases where the covariance matrix is close to unit is for the // balsa during measurements in the vacuum and with natural convection. It is probably because the $z$-positions were not well-chosen; move them would help to get a better covariance and a stronger estimation. Finally, the estimated heat exchange coefficients seem consistent with the type of environment condition, even if results are rather scattered and independently of the estimated thermal diffusivity values.

Table 2: Estimation results for the five samples.

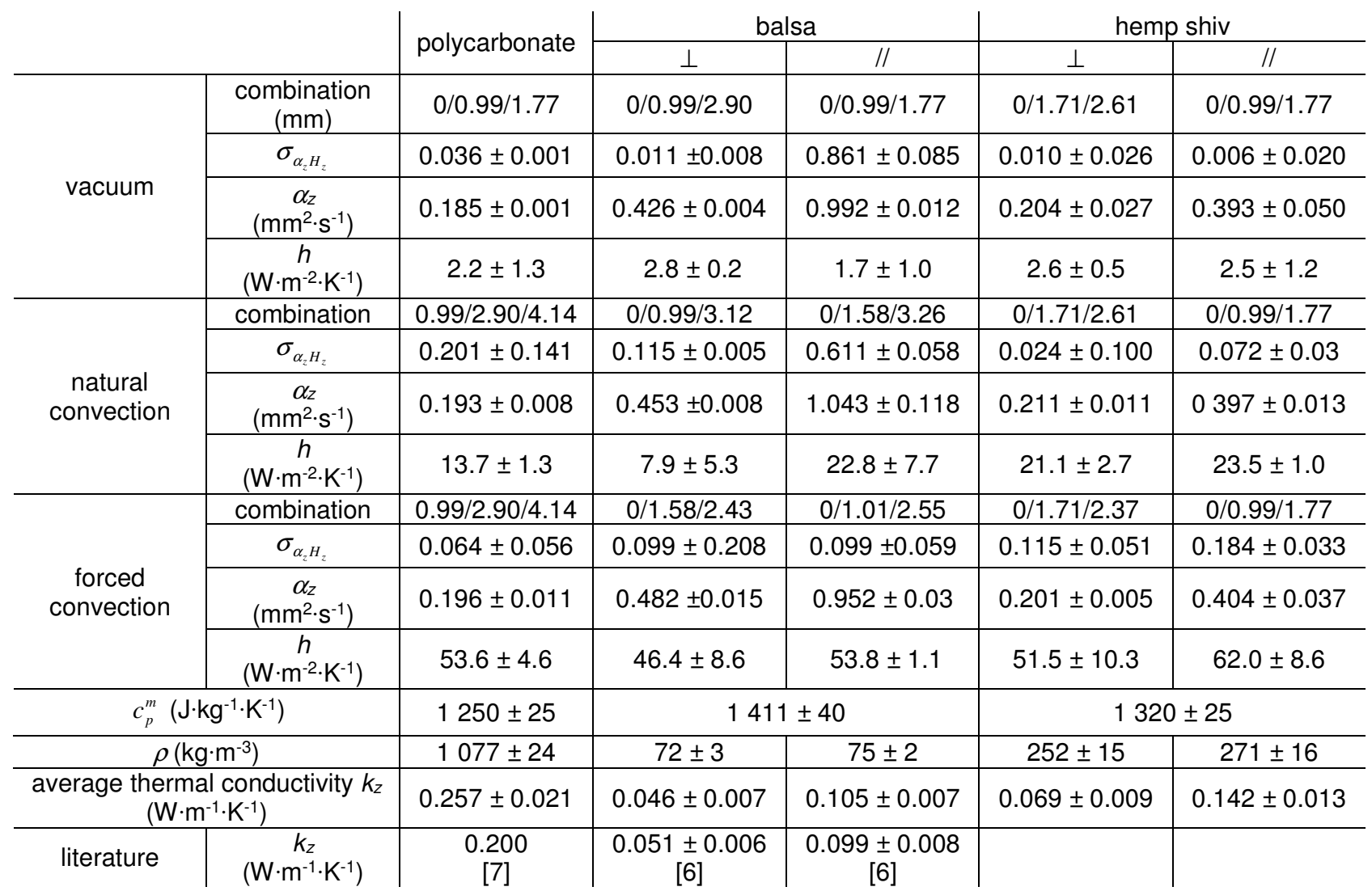




\section{Conclusion}

This study presented a methodology to estimate thermal diffusivity of a low conductivity millimeter-sized material, the hemp shiv, mainly used in the fabrication of hemp concrete. The method was initially applied on well-known materials, polycarbonate and balsa. Experimentally, the samples were heated on one side during few seconds and the evolution of the temperature field of the samples surface was measured by an IR camera. Three thermograms at three locations $z_{1}, z_{\text {, }}$ and $z_{2}$ were then used to perform the estimation of the thermal diffusivity and also of a modified Biot number, since the theoretical model is based on the fin effect. The influence of the environment was studied, since three types of surrounding environments were possible: vacuum, natural convection, and forced convection. Estimation results were in good agreement with literature regarding the polycarbonate and the balsa. The parameter estimation with the hemp shiv has given results consistent with the thermal properties of cellulosic materials. Several criteria have been analyzed in order to verify the feasibility and the quality of the estimation, such as the location of thermograms, the reduced sensitivity of the temperature with both the diffusivity and the modified Biot number, the covariance matrix, and the residuals. The influence of the orientation of the samples fibers for the balsa and the hemp shiv with the heat flux has shown a ratio of about 2 on the thermal diffusivity, and thus on the conductivity.

\section{REFERENCES}

[1] T. Pierre, T. Colinart, P. Glouannec, Measurement of thermal properties of biosourced building materials, Int J Thermophys (2014) 35:1832-1852.

[2] S. T. Nguyen, A. D. Tran-Le, M. N. Vu, Q. D. To, O. Douzane, T. Langlet, Modeling thermal conductivity of hemp insulation material: A multiscale homogenization approach, Building and Environment 107 (2016) 127-134.

[3] J. Sjöström, P. Blomqvist, Direct measurement of the thermal properties of wood pellets: Elevated temperatures, fine fractions and moisture content, Fuel 134 (2014) 460-466.

[4] B. Rémy, A. Degiovanni, D. Maillet, Measurement of the in-plane thermal diffusivity of materials by infrared thermography, International Journal of Thermophysics, Vol. 26, No. 2, March 2005.

[5] D. Maillet, S. André,J.-C. Batsale, A. Degiovanni, C. Moyne, Thermal quadrupoles, Solving the heat equation through integral transforms, John Wiley \& Sons, Ltd., New-York, 2000.

[6] P. Carré, R. Le Gall, Définition et détermination des conductivités thermiques dans les structures C.V.R. - balsa, Rev. Gén. Therm. Fr., n³40, p. 211-215, avril 1990.

[7] J. G. Speight, N. A. Lange, Lange's handbook of chemistry, McGraw-Hill 2005, 16 éd., p. 2.758, 2005.

[8] A. Missenard, Conductivité thermique des solides, liquides, gaz et de leurs mélanges, Editions Eyrolles, Paris, 1965. 